\title{
A Novel Noncooperative Behavior Management Method for Multiattribute Large Group Decision-Making
}

\author{
Xiaoqin Dong, ${ }^{1,2}$ Ying Yang, ${ }^{1}$ Bo Shao $\left(D,{ }^{1}\right.$ and Xianbin Sun ${ }^{2}$ \\ ${ }^{1}$ College of Hydraulic \& Environmental Engineering, China Three Gorges University, Yichang 443002, China \\ ${ }^{2}$ School of Civil Architecture and Environment, Hubei University of Technology, Wuhan 430068, China \\ Correspondence should be addressed to Bo Shao; shaobo910@whu.edu.cn
}

Received 25 December 2021; Accepted 11 January 2022; Published 14 February 2022

Academic Editor: Huihua Chen

Copyright ( $(2022$ Xiaoqin Dong et al. This is an open access article distributed under the Creative Commons Attribution License, which permits unrestricted use, distribution, and reproduction in any medium, provided the original work is properly cited.

In multiattribute large-group decision-making (MALGDM), the ideal state indicates a high degree of consensus for decisionmakers. However, it is difficult to reach a consensus because the conflict between various decision attributes and decision-makers increases. To deal with the problem, a novel consensus model was developed to manage the decision-making in large groups based on noncooperative behavior. The improved clustering method was used to take account of the similarities among different decision-makers, while similar decision-makers will be grouped into the same group. Moreover, the consensus threshold was determined from an objective and subjective aspect to judge whether the consensus reaching process continues. The noncooperative behavior and adjustment amount of decision-makers' opinions were investigated based on the proposed consensus model, and an emergency decision-making problem in flood disaster is applied to manifest the feasibility and distinctive features of the proposed method. The results show the proposed novel consensus model demonstrated strong applicability and reliability to the noncooperative subgroup problem and can be explored to manage multiattribute interactions in LGDM.

\section{Introduction}

Decision-making, which aims at identifying an ideal alternative based on the information described by decisionmakers, is widely used in all aspects of modern life [1-3]. With the increase in the complexity of decision-making problems, many attributes relevant to decision-making problems have been explored [4]. Decision-makers need to consider all relevant aspects of the problem [5]. The decision-making behavior of decision-makers depends on many factors, including their personal and professional goals, interests, and the experience they pursue to develop themselves professionally [6]. Decision-makers need to know about Business, Management and Accounting, Engineering, Social Sciences, and Computer Science to inform the decision-making process [7]. Group decision-making (GDM) has attracted increasing attention due to its characteristic superiority of gathering knowledge of decisionmakers from various fields [8-10]. Problems always involve many interconnected fields, and the decision-making results are related to the benefits of stakeholders. Thus, it becomes uneasy for small-group decision-making to reach the demands of social development $[11,12]$. As the number of people in the decision-making group increases, the problem of multiattribute large group decision-making appears [13].

Clustering processing, the essential part of large group decision-making, is a fundamental but indispensable process in multiattribute large-group decision-making (MALGDM) [14]. The clustering process in MALGDM can improve the efficiency of the decision process [15]. The C-means algorithm [16] and the k-means algorithm [17] are the most available clustering methods in applications. Xue used the Choquet integral (CI) operator to measure each attribute and then aggregated it [18]. Based on Shannon entropy, Li measured the uncertainty of discrete Z-numbers by a new technique [19]. The main features of these algorithms are the early decided clustering numbers and the effect of threshold selection on classification results. Having defined the shape similarity measure, Tapia-Rosero et al. developed a clustering method, in which similar-shaped membership functions are grouped by agglomerative hierarchical clustering technique [20]. However, this method did not 
consider the similarity of decision-makers in the same cluster, and there exist errors for the shape similarity measure [21]. Besides, a clustering method based on vector space was proposed by $\mathrm{Xu}$ et al. This method considered the similarity of decision-makers' decisions [22, 23]. However, these methods classified the cluster by calculating the similarities between the decision-makers and the cluster. But those methods did not consider the similarities between two decision-makers in the same clusters. The similarities between decision-makers will decrease as the clustering processes develop.

The consensus level is selected to describe decisionmakers' differences in opinion and preference. It can also measure the degree of agreement among different clusters in MALGDM [24]. There are many approaches to compute the consensus level [25-27]. In MALGDM, before making a group decision, a consensus reaching process is usually applied to reach the collective agreement $[28,29]$. To reach a consensus, some decision-makers must modify their opinions in the dynamic and iterative group discussions [30]. For reaching a consensus process in MALGDM, many scholars do a lot of studies. Pérez et al. introduced a feedback mechanism and built a consensus model based on decisionmakers' relevance or importance level [31]. For MALGDM problems, $\mathrm{Xu}$ et al. promoted the consensus reaching process in two stages. The novel method could appropriately adjust the preference of decision-makers and the weight of subgroups [32]. Jin et al. adopted a local adjustment strategy to retain preference evaluation of decision-makers as far as possible in group decision-making [33]. To reach consensus, $\mathrm{Xu}$ et al. proposed a dynamic consensus method based on an exit authorization mechanism. They thought the subgroup would be suggested to exit the decision-making process once the proximity index could not meet the requirement, and then the delegation mechanism is employed to reserve the cluster's influence by giving trust weights to other clusters [24]. Noncooperative behavior is common in the practical process of decision-making. However, these studies are lacking at considering the influence of noncooperative behavior. To reach a consensus, a suitable method should be adopted to manage the noncooperative behavior. Based on a self-management mechanism of noncooperative behaviors, Dong et al. introduced three kinds of noncooperative behaviors and developed a new consensus framework [34]. They also proposed a novel framework based on a selfmanagement mechanism for noncooperative behaviors in large-scale consensus reaching processes [35]. Palomares provided a consensus model suitable to handle and detect the noncooperative behavior, and the consensus could be reached by decreasing the weight of noncooperative cluster [36]. $\mathrm{Wu}$ and $\mathrm{Xu}$ proposed a consensus model, in which the clusters can be changed. The clusters can modify if the individuals are able to change their preferences via the consensus reaching process [37]. Quesada et al. introduced a methodology to process noncooperative behaviors, in which a uniform-based weighting scheme was adopted to compute the weight of decision-makers in LGDM [38]. Nazari et al. used dynamic noncooperative games to model these conflicts when stakeholders appear noncooperative behavior
[39]. With the purpose of managing minority opinion and noncooperative behavior in MALGDM, Xu and put forward the concept of comprehensive adjustment coefficient and designed an improved consensus model [40].

To sum up, problems have evolved into many interrelated areas, and the decision-making of large groups needed to meet the requirements of social development. But regarding LGDM, it is not easy to reach the consensus level in decision processes. The goal of large group decision-making is to find a method that can reach consensus effectively in large groups, improve the consensus level in short time, and obtain the accurate decision-making result. In the previous literature, the subgroup obtained in these studies remains unchanged in the consensus reaching process. This is often untrue because decision-makers modify their available opinion. The subgroup opinion must be changed when decision-makers modify their opinion. This will lead to unacceptable results of large group decision making. Meanwhile, the decision-makers in the noncooperative subgroup who are willing to modify their decision opinion should not be penalized. Unlike previous literature, this paper protected individual decision opinion. And previous methods did not consider the similarity of decision-makers in the same cluster. This will reduce the consistency of the group. Mandal et al. acknowledged noncooperative behaviors and divided them with the experts' similar evaluations into a subgroup. But decision-makers were still unable to change subgroup [41]. Therefore, it is necessary to seek a large group decision-making method that can protect the opinions of decision-makers and improve the degree of consensus. The main contribution of the paper is a precise consensus reaching model that we proposed to manage noncooperative behaviors in MALGDM. And an improved clustering method is developed. This method considers the similarities among decision-makers.

The remaining part of the paper is structured as follows: firstly, decision-makers are clustered, and the group consensus level is obtained in Section 2. In Section 3, the noncooperative behavior is detected and managed. A typical example, applied to indicate the utility and applicability of this model, is shown in Section 4. Then, Section 5 discusses the advantages and innovations of the proposed methods in detail. Finally, conclusion and future researches are provided in Section 6.

\section{Preliminaries}

2.1. Problem Formulation. MALGDM problems can be defined as a situation where a large number of decisionmakers must make a high-quality decision result by choosing the $n$ alternatives. The main parameters of MALGDM are as follows:

(1) A discrete finite set of alternatives $x=\left\{x_{1}, x_{2}, \cdots x_{n}\right\}$, where $x_{i}$ represents alternative solution.

(2) A set of decision-makers can be denoted as $E=\left\{e_{1}, e_{2} \ldots e_{M}\right\}(M \geq 2)$. The weight vector of decision-makers is $\omega=\left\{\omega_{1}, \omega_{2}, \cdots \omega_{M}\right\}$, where $\omega_{j}(j=$ $1,2 \ldots M) \in(0,1)$ and $\sum_{j=1}^{M} \omega_{j}=1$. After collecting a 
lot of previous literature, we can determine the range of the number of large groups of decision makers. Usually, when the number of experts in a group reaches 20 , that is, $M \geq 20$, the group is considered as a large group. And the decision-making process in which they participate can be defined as large group decision-making $[42,43]$.

(3) A finite set of attributes $F=\left\{f_{1}, f_{2} \ldots f_{P}\right\}(P \geq 2)$, the weight vector of attribute $\eta=\left\{\eta_{1}, \eta_{2}, \cdots \eta_{P}\right\}$, where $\eta_{k} \geq 0(k=1,2, \ldots P)$, and $\sum_{k=1}^{P} \eta_{k}=1$.

Each decision-maker will give a numerical decision matrix to express the opinion for the alternatives $A^{j}=\left(a_{i k}^{j}\right)_{n \times P}, j \in M$, where $a_{i k}^{j}$ represents decision-maker $e_{j}$ 's preference of alternative $x_{i}$ concerning attribute $f_{k}$. The premise of this paper is that there is disagreement among decision-makers in the group. So, it is not possible for all decision-makers to behave the same preference for $x_{i}$. This represents that $\max _{i}\left\{a_{i k}^{j}\right\} \neq \min _{i}\left\{a_{i k}^{j}\right\}$. Different attributes in MALGDM problems are often measured in different units, so the preference value should decision matrix $V^{j}=\left(v_{i k}^{j}\right)_{n \times P}$ as follows:

$$
v_{i k}^{j}=\frac{a_{i k}^{j}-\min _{i}\left\{a_{i k}^{j}\right\}}{\max _{i}\left\{a_{i k}^{j}\right\}-\min _{i}\left\{a_{i k}^{j}\right\}} .
$$

2.2. Clustering Method for Large-Group Members. To simplify the decision-making processes, decision-makers are clustered by individual decision matrix $A^{j}$ to transform into small-group decision making in LGDM. decision-makers are clustered into $l$ clusters $(1<Y<n)$ by the improved mean of preference clustering method, which is described in Algorithm 1.

2.3. Determination of Consensus Level. Decision-makers' respective weights in the decision processes can be determined by the following definitions:

Definition 1. Experts in larger subgroups should be given larger weights based on the majority principle [37]. In line with the number and weight values of experts in the subgroup, the subgroup weight value can be defined as

$$
\lambda_{l}=\frac{\left(\sum_{j=1}^{M} \omega_{j} \times \theta_{j l}\right)^{2}}{\sum_{l=1}^{Y}\left(\sum_{j=1}^{M} \omega_{j} \times \theta_{j l}\right)^{2}},
$$

where $\theta_{j l}=1$ represents expert $e_{j}$ belonging to the subgroup $C^{l} ; \theta_{j l}=0$ represents expert $e_{j}$ not belonging to the subgroup $C^{l}$, where $l=1,2, \ldots, L$, and it is easy to know that $0 \leq \lambda_{l} \leq 1$ and $\sum_{l=1}^{Y} \lambda_{l}=1$.

Definition 2. An individual decision matrix can be obtained by decision-makers' opinions, the subgroup decision matrix is computed by aggregating the individual matrices, considering the weights associated with each decision-maker, and the subgroup decision matrix can be calculated [44].

$$
r_{i k}^{l}=\sum_{j=1}^{M} \omega_{j} \times v_{i k}^{j} \times \theta_{j l} .
$$

By aggregating single subgroup decision matrixes, the normalized group decision matrix $R^{c}=\left(r_{i k}^{c}\right)_{n \times P}$ is obtained, where $r_{i k}^{c}$ is represented as

$$
r_{i k}^{c}=\sum_{l=1}^{Y} \lambda_{l} \times r_{i k}^{l}
$$

Definition 3. According to the gap subgroup decision matrix $R^{l}(l=1,2, \cdots, Y)$ and the group decision matrix $R^{c}$, the consensus level $C I\left(R^{l}\right)$ between the subgroups' decision matrices $R^{l}(l=1,2, \ldots, Y)$ and the group decision matrix $R^{c}$ is defined as

$$
C I\left(R^{l}\right)=1-d\left(R^{l}, R^{c}\right)
$$

where $d\left(R^{l}, R^{c}\right)$ is the Manhattan distance between $R^{l}$ and $R^{c}$; that is, $d\left(R^{l}, R^{c}\right)$ represents the similarity between subgroup $C^{l}$ and $C^{C}$, which can be defined as

$$
d\left(R^{l}, R^{c}\right)=\frac{1}{n} \times \sum_{i=1}^{n} \sum_{k=1}^{P} \eta_{k} \times\left|r_{i k}^{l}-r_{i k}^{c}\right| .
$$

By calculating the average value of consensus level $C I\left(R^{l}\right)$, the group consensus level LGCI can be obtained:

$$
\mathrm{LGCI}=\lambda_{l} \times \sum_{l=1}^{Y} C I\left(R^{l}\right) .
$$

If $L G C I=1$, it indicates that all decision-makers have reached consensus among the large groups. A $\overline{L G C I}$ indicates a higher level of consensus among all decision-makers. $\overline{L G C I}$, as the group consensus threshold, is set to determine whether the consensus reaching process should be carried out. If $L G C I \leq \overline{L G C I}$, the consensus process should be used to change decision-makers' opinions to reach a higher consensus level.

2.4. Determination of Consensus Threshold. The consensus threshold $\overline{L G C I}$ should be determined to judge whether the consensus reaching process continues or not. The decisionmaking pressure of large groups often leads to the uncertainty and subjectivity of the opinion adjustment coefficient. In order to improve the accuracy of group decision making, both objective and subjective factors should be considered. And a coefficient should be set to adjust the subjective factors and objective factors.

2.4.1. Subjective Threshold. For the particular problem, the decision-makers provide the consensus threshold $\overline{L G C I}$ according to the quality of the problem [45]. The consensus threshold $\overline{L G C I}$ reflects its attitude towards the group consensus level and opinion. If the consequence of the decision is important, the consensus threshold should be as high as possible. In this article, let the subjective consensus 
Input: the individual matrix $A^{j}$ and the threshold $\zeta$, which is determined according to practical decision situation.

Output: the number of subgroup $l$ and the subgroups $C_{1}, C_{2}, \ldots C_{Y}$.

Step 1. Construct the decision set $U$ comprising all individual decision matrix, that is, $U=\left\{V^{1}, V^{2}, \ldots V^{M}\right\}$.

Step 2. Initialize $Y=1$ as the number of subgroups, and select decision-maker $e_{1}$ as the member of this subgroup, this subgroup is defined as $C^{l}$, and $S$ stands for a temporary set.

Step 3. Select decision-maker $e_{q}$ sequentially from the set $E$ and allocate them to the subgroup $C^{l}$. Then remove the decision matrix from the set $U$ and let the number of members in subgroup $C^{l}$ be $z_{l}$.

Step 4. Compute the gather degree between decision-maker $e_{q}$ and each subgroup $C^{l}$, which can be denoted as:

$$
\mu_{j l}=1-\underset{\substack{q=1 \\ z_{l}}}{\max } 1 / n \sum_{i=1}^{n} \sum_{k=1}^{P} \eta_{k} \times\left|v_{i k}^{j}-r_{i k}^{q}\right|\left(j \neq q, e_{q} \in C^{l}, l \in Y\right)
$$

where, $z_{l}$ is the number of decision-maker in the subgroup $C^{l}, \mu_{j l}$ represents the gather degree of decision-makers $e_{j}$ in the subgroup

$C^{l}$, if the $\mu_{j l} \geq \zeta$, decision-maker $e_{j}$ is allocated to the subgroup $C^{l}$, and remove it out from the set $U$, let $z_{l}=z_{l}+1$. Otherwise, decision-maker $e_{j}$ is allocated to the temporary set $S$.

Step 5. If $S$ is not null, let $C^{L+1}=S, T=$ null and $Y=Y+1$ respectively, and get back to Step 3; otherwise, go to Step 6 .

Step 6. Record the results of clustering. The number of subgroups is recorded as $Y$, and the number of members is recorded as $z_{l}$ in the subgroup $C^{l}$.

\section{Algorithm 1:}

threshold determined by the decision-makers' experience be $\overline{L G C I}_{s u b}$. The subjective consensus threshold is the decisionmaker's expected consensus level.

\subsubsection{Objective Threshold. The objective consensus} threshold $\overline{L G C I}_{o b}$ is determined to improve the feasibility of the consensus reaching process. The objective consensus threshold $\overline{L G C I}_{o b}$ represents that the large group can reach consensus level by the original decision matrices of decisionmakers, which is defined as

$$
\overline{\mathrm{LGCI}}_{o b}=\mathrm{LGCI} \text {. }
$$

Definition 4. The consensus threshold should satisfy two conditions: one is that the consensus threshold meets the requirement of practical decision problem, which is described as subjective consensus threshold; the other is that the consensus threshold can be reached in the opinion adjustment scale, in which the decision-maker is willing to modify, which is defined as the objective consensus threshold. Thus, it is feasible to determine the consensus threshold by the objective consensus threshold and the subjective consensus threshold:

$$
\overline{\mathrm{LGCI}}=\alpha \overline{\mathrm{LGCI}}_{o b}+(1-\alpha) \overline{\mathrm{LGCI}}_{\mathrm{sub}},
$$

where $\alpha$ is the consensus threshold adjustment coefficient and defined by the number and weight of decision-makers in the subgroup whose consensus level is less than the group consensus level calculated by the initial group decision matrix.

Definition 5. Let the subgroup set where consensus level is more than the group consensus level be $G$, which is described as

$$
G=\left\{C^{l} \mid C I\left(R^{l}\right)>\mathrm{LGCI}\right\} .
$$

The adjustment coefficient $\alpha$ can be calculated as

$$
\alpha=\frac{\sum_{\lambda=1}^{Y} \lambda_{l} \times \vartheta_{l g}}{\sum_{l=1}^{Y} \lambda_{l}} .
$$

where $\vartheta_{l g}$ represents the subgroup $l$ belonging to $G$.

\section{Process of Noncooperative Behaviors}

In this section, a consensus reaching model suitable for addressing noncooperative behavior in MAGDM problems is proposed. The innovative point of this model features in the abilities to detect and handle individual and subgroup noncooperative behaviors in the consensus reaching process, with the aim of improving the overall consensus reaching process performance.

3.1. Noncooperative Behavior Detection. In the consensus reaching model presented in this study, we define an approach to identify those noncooperative subgroups that exist as decision-makers who are reluctant to change their original preferences to reach a consensus, which is aiming at assisting the subsequent treatment of such decision-makers, so as to improve the performance of the consensus reaching process. The detection approach is first used in the second round of the consensus reaching process, because of its requirement of comparisons among subgroups obtained in the previous and current rounds of discussion. There exist three rules to detect the noncooperative subgroup according to the definition of noncooperative behavior. Let the noncooperative subgroup be $C^{l^{*}}$. The detection method includes two steps:

\section{Step 1. Determine the detection object}

Before the noncooperative subgroup is detected, the detection object should be determined. The noncooperative subgroup includes two common characteristics according to the definition:

(i) The consensus level of the subgroup opinion is smallest, which can be denoted as 


$$
C^{l^{*}}=\stackrel{\substack{l=1 \\ Y}}{\min }\left\{C I\left(R^{l(t-1)}\right) \mid l=1,2, \ldots, Y\right\} .
$$

(ii) The number of decision-makers in these subgroups is small, in a general way, which is less than $[\mathrm{M} / n]$, described by the following formula:

$$
\sum_{j=1}^{M} \theta_{j l^{*}}^{t-1} \leq \frac{M}{Y}
$$

For example, a large group consists of 15 decision makers, and there are three decision options. The number of people supported by the three decision schemes is 7, 5, and 3, respectively. Their CI value size relation is $\mathrm{CI}_{1}>\mathrm{CI}_{2}>\mathrm{CI}_{3}$. According to characteristic (i), we can know that the third subgroup may be a noncooperative group. We can know that $[\mathrm{M} / n]$ is 5 , the third group's number is 3 , and 3 is smaller than 5. So, according to characteristic (ii), the third subgroup can be determined by the detection object.

Step 2. Detect the noncooperative subgroup

After the detection object is determined, the subgroup $C^{l^{*}}$ will adjust their decision opinions by discussion in the $t-1$-th round, and the adjustment decision matrix of decision-maker $A^{j(t)}$ in the subgroup $C^{*^{*}}$ is obtained, and the temporary subgroup decision matrices $R^{\prime l(t)}$, in which the form of subgroup is not changed, are calculated. For the noncooperative subgroup, there exists at least one decision-maker in the subgroup reluctant to adjust their own opinions, which is checked to determine whether decision-maker in the subgroup $C^{l^{*}}$ is willing to adjust their opinions or not. To do this, the temporary subgroup consensus level $C I^{\prime}\left(R^{l(t)}\right)$ and the temporary consensus level $L G C I^{\prime(t)}$ can be calculated by the temporary subgroup decision matrices $a^{\prime l(t)}$. Thus, there are three situations to define the noncooperative subgroup:

(i) Decision-makers in $C^{l^{*}}$ are willing to adjust their opinions, but the decision opinion adjustment of decision-maker causes negative influence to the consensus level of $C^{l^{*}}$ :

$$
\begin{aligned}
C^{l^{*}}= & \left\{C^{l} \mid C I^{\prime}\left(R^{l(t)}\right)\right. \\
& \left.<C I\left(R^{l(t-1)}\right) ; l=1,2, \ldots, Y\right\} .
\end{aligned}
$$

(ii) Decision-makers in $C^{l^{*}}$ do not change their opinions, that is, the consensus level of $C^{*^{*}}$ in $t$-th round keeping correspondence with $t$-1-th round:

$$
\begin{aligned}
C^{l^{*}} & =\left\{C^{l} \mid C I^{\prime}\left(R^{l(t)}\right)\right. \\
& \left.=C I\left(R^{l(t-1)}\right) ; \quad l=1,2, \ldots, Y\right\} .
\end{aligned}
$$

(iii) The decision opinion adjustment of decision-maker causes positive influence to the consensus level of $C^{l^{*}}$, but there are only a small part of decision- makers transforming their opinions, and there exist decision-makers who are not willing to modify their opinions. The noncooperative subgroup detection rule can be expressed as

$$
C^{l^{*}}=\left\{C^{l} \mid C I^{\prime}\left(R^{l(t)}\right)>C I\left(R^{l(t-1)}\right) ; \sum_{j=1}^{n_{l}} \sigma_{j l} \neq z_{l} l=1,2, \ldots, Y\right\} .
$$

In the example in step (1), there are three decision-makers in the detection subgroup. If the subgroup has noncooperative behavior, then at least one person did not change his preference, or the decision opinion adjustment of decision-maker causes negative influence to the consensus level of $C^{l^{*}}$. If all decisionmakers adjust their opinions, but $C I^{\prime}\left(R^{l(t)}\right)<C I\left(R^{l(t-1)}\right)$, this is the first situation of noncooperative subgroup. If all decisionmakers do not adjust their opinions, this is second situation. If at least one decision-maker did not adjust his opinion, despite the adjustments made by the rest of the decision-makers, and those adjustments have had a positive impact to the consensus level, subgroups are also considered as noncooperative subgroup. This is the third situation.

where $\sigma_{j l}$ represents decision-maker $e_{j}$ willing to change their opinions, and $\sigma_{j l}$ is detected by change degree. The change degree is introduced to measure the decision-maker who modify his opinion [32]; it can be described as

$$
\varphi_{j l}^{t}=\frac{1}{n} \sum_{i=1}^{n} \sum_{k=1}^{P} \eta_{k} \times\left|v_{i k}^{j(t)}-r_{i k}^{l(t-1)}\right| \times \theta_{j l}^{t}
$$

$\sigma_{j l}$ can be computed by comparing the change degree between $t$ - 1 -th round and $t$-th round, which is defined as

$$
\sigma_{j l}= \begin{cases}1, & \text { if } \varphi_{j l}^{t}-\varphi_{j l}^{t-1} \neq 0 \\ 0, & \text { if } \varphi_{j l}^{t}-\varphi_{j l}^{t-1}=0\end{cases}
$$

\subsection{Management Strategy of the Noncooperative Behavior.} Through Step 3.1, we identified three different types of noncooperative behavior. But the core of this paper is to develop different strategies for different noncooperative behaviors. Thus, unlike the traditional adjustment method for noncooperative behaviors, a new strategy was devised in this study. The management strategy is determined by analyzing the noncooperative degree of noncooperative subgroup. And the noncooperative subgroup is allowed to change. The consensus level needs to be recalculated after the subgroup changed. The concrete process of management strategy is described as follows:

Step 1: measure the noncooperative degree

For the noncooperative subgroup, the degree of noncooperation is used to describe the decisionmaker's willingness to change the decision to improve group consensus level. Thus, the degree of noncooperation is influenced by two factors: one is the number of decision-makers who have changed their views, and the other is whether the changed opinion can increase the level of consensus. 
The number of policymakers who changed their minds first needs to be counted. In general, the LGCI can reflect whether the noncooperative subgroup modify their initial opinions or not, and the number of decision-makers who are willing to modify their opinions describe the adjustment degree of noncooperative subgroup, which is expressed as

$$
\gamma_{l^{*}}^{t}=\sum_{j=1}^{M} \sigma_{j l^{*}} \times \theta_{j l^{*}}
$$

And then, Thus, the change of LGCI value can indicate whether a change in decision-makers' opinion has increased the level of group consensus. Thus, the noncooperative degree is measured by the value of $L G C I$ and the number of decision-makers who are willing to modify their opinions, which is obtained as follows:

$$
\phi_{l^{*}}^{(t)}=\frac{\left(\mathrm{LGCI}^{t}-\mathrm{LGDI}^{t-1}\right) \gamma_{l^{*}}^{t}}{\left|\mathrm{LGCI}^{t}-\mathrm{LGDI}^{t-1}\right| z_{l^{*}}} .
$$

$\phi_{l^{*}}^{(t)}$ can represent the degree of noncooperation, and the number of experts in the MALGDM is odd, so $\phi_{l^{*}}^{(t)}$ is not equal to 0.5 ; there are three cases according to the calculation results.

(i) If $\phi_{l^{*}}^{(t)} \leq 0$, it means that the subgroup $C^{l^{*}}$ manifests a very high degree of noncooperative behavior. A small number of decision-makers in noncooperative subgroup will change their opinions in the $t$ - 1 th round, and the changed result is negative for the consensus level.

(ii) If $0<\phi_{l^{*}}^{(t)}<0.5$, the subgroup $C^{l^{*}}$ is considered as a partly noncooperative subgroup. A small part of the decision-maker in noncooperative subgroup modified their opinions in the $t$-1-th round, and the change result of subgroup is positive for consensus level.

(iii) If $0.5<\phi_{l^{*}}^{(t)} \leq 1$, it indicates that the subgroup $C^{l^{*}}$ is a cooperative subgroup, and more than half of decision-makers in the subgroup $C^{l^{*}}$ change their opinions, and the change result of subgroup is positive for consensus level.

Step 2: process the noncooperative behavior When the subgroup's noncooperative degree is got, the novel noncooperative behavior treatment method that considers the change of subgroup is developed. The following strategies are adopted for the three cases with different degrees of noncooperation.

(i) For the noncooperative subgroup, that is, $\phi_{l^{*}}^{(t)} \leq 0$, the decision-makers in subgroup $C^{l^{*}}$ not only do not improve the group consensus, but also may cause the group consensus decrease. To speed up the decision-making processes and obtain proper results with a short period of time, the subgroup $C^{l^{*}}$ will be suggested to withdraw from the decision process. (ii) For the partly noncooperative subgroup, that is, $0<\phi_{l^{*}}^{(t)}<0.5$, a major part of decision-makers do not change their opinions in the subgroup $C^{l^{*}}$. It should be penalized by adjusting the weight of subgroup. But there are minor decision-makers willing to modify their decision opinion, and their opinions should be protected. Therefore, we need to determine whether the decision-maker who changes perspective belongs to the subgroup $C^{l^{*}}$. And then the form of subgroup $C^{l^{*}}$ may be changed. Thus, the treatment method is determined by judging whether the decision-makers belong to the subgroup $C^{l^{*}}$.

Let the set of decision-makers who are unwilling to modify his opinion be $C^{\prime l^{*}}$ in the subgroup $C^{l^{*}}$, and the number is $z_{l^{*}}$, the set of decision-makers who are willing to modify his opinion is $B^{l^{*}}$. That is, $B^{l^{*}}=\left\{e_{j^{*}} \mid e_{j^{*}} \in C^{l^{*}}, e_{j^{*}}\right.$ $\left.\notin C^{l^{*}}\right\}$. Based on the (17), we proposed a method to judge whether the decision-maker belongs to the subgroup $C^{l^{*}}$, which is denoted as

$$
\begin{aligned}
& \varphi_{j^{*} l^{*}}^{{ }^{\prime}=} \underset{\substack{q^{*}=1 \\
l^{*}}}{\max } \frac{1}{n} \sum_{i=1}^{n} \sum_{k=1}^{P} \eta_{k} \times \sigma_{j^{*} l^{*}}^{t} \times\left|v_{i k}^{j^{*} t}-r_{i k}^{q^{*} t}\right| \\
& \cdot\left(j^{*} \neq q^{*}, e_{q^{*}} \in C^{\prime} l^{*}, l^{*} \in Y\right) .
\end{aligned}
$$

where $\varphi_{j^{*} l^{*}}^{t}$ represents the conflict degree between $e_{j^{*}}$ and subgroup $C^{l^{*}}$ in the $t$-th round.

If $\varphi_{j^{*} l^{*}}^{t} \leq 1-\varsigma$, it represents that the decision-maker $e_{j^{*}}$ belongs to the subgroup $C^{l^{*}}$ after his opinion is changed.

If $\varphi_{j^{*} l^{*}}^{t}>1-\varsigma$, it represents that the decision-maker $e_{j^{*}}$ does not belong to the subgroup $C^{l^{*}}$ after his opinion is changed, and the decision-maker $e_{j^{*}}$ can be clustered based on the procedure in Algorithm 1.

Although there are a little of decision-makers to modify their opinions in partly noncooperative subgroup, the subgroup $C^{l^{*}}$ still expresses the lower cooperative level. Thus, in order to reduce its impact on the group consensus level, the weight of subgroup $C^{l^{*}}$ also needs appropriate adjustment. The modified function is a decreasing function. To describe the interaction between the weight adjustment and the number of decision-makers, the weight adjustment function is developed based on the number of decisionmakers who is willing to modify its own opinion, which is defined as

$$
\lambda^{\prime} l^{*}{ }_{t}=\frac{z_{l^{*}}^{t}-z^{\prime} l^{*}{ }_{t}}{z^{\prime} l^{*}{ }_{t}} \lambda_{l^{*}}^{t}
$$

where $\lambda_{l^{*}}^{\prime}$ is the weight of subgroup $C^{l^{*}}$ after adjustment in the $t$-th round, and $\lambda_{l^{*}}^{t}$ is the weight of subgroup $C^{l^{*}}$ in the $t$ th round. Generally speaking, subgroup weights reflect their contributions to the group consensus level. When the opinions of decision-makers in the subgroup $C^{l^{*}}$ are modified, the subgroup' contributions are adjusted too. The greater the contribution of subgroup to consensus level, the more important it is. Individuals who have changed opinion 
with negative effect to the group consensus level should reduce some weight [46]. Based on this rule, the contributions of the subgroup are introduced to measure the subgroup weight in the $t$-th round, which can be defined as

$$
D_{l^{*}}^{t}=\mathrm{LGCI}^{t}-\mathrm{LGCI}_{l^{*}}^{t} \text {. }
$$

where $D_{l^{*}}^{t}$ represents the contributions of subgroup $C^{l^{*}}$ for the group consensus level. $L G C I_{l^{*}}^{t}$ denotes the group consensus level without the subgroup $C^{*^{*}}$ in the $t$-th round, which is defined as

$$
\operatorname{LGCI}_{l^{*}}^{t}=\sum_{l=1, l \neq l^{*}}^{L} \lambda_{l}^{t-1} \sum_{i=1}^{n} \frac{1}{n} \sum_{k=1}^{P} \eta_{k} \times\left|r_{i k}^{l(t)}-r_{i k}^{c(t)}\right| .
$$

To protect the interest of decision-makers who are willing to modify its own opinion, we need to update the weights of the subgroup and recalculate the group-decision consensus level. The following equations show how to update the weights:

$$
\lambda_{l^{*}}^{t}=\lambda_{l^{*}}^{t-1} \times\left(1+D_{l^{*}}^{t}\right)^{\xi}
$$

$\xi$ expresses the impact of the subgroups' contributions on their weights, which are usually given by the decision-makers in advance. If the decision problem is in urgency and has to be dealt with in time, it should be assigned less restrictive values. Otherwise, more restrictive values must be put into use.

(iii) For the cooperative subgroup, that is, $0.5<\phi_{l^{*}}^{(t)}<1$, more than half of decision-makers in this subgroup are willing to modify their initial opinions. This subgroup expresses a very high degree of cooperative behavior. Thus, the motivation mechanism should be adopted to protect the decision opinion of this subgroup. Similar to partly noncooperative subgroup, if the decision-maker $e_{j^{*}}$ does not belong to the subgroup $C^{l^{*}}$ after his opinion is changed, Algorithm 1 can be used to cluster the decisionmaker $e_{j^{*}}$ into a suitable subgroup. If the decisionmaker $e_{j^{*}}$ belongs to the subgroup $C^{l^{*}}$ after his opinion is changed, the motivation mechanism is similar to the treatment process of partly noncooperative subgroup. When the number of decisionmakers who modify their own opinions is more than the half of subgroup, the adjustment coefficient of the subgroup in equation (26) is not less than 1 . Thus, the weight of subgroup will be enhanced.

3.3. Algorithm of Large Group Consensus. Adopting consensus reaching model that the basic thought is to adjust decision information matrix, to enable the decision-makers to have a higher consensus level, the noncooperative subgroup is detected and addressed to obtain a higher consensus level. Algorithm 2 of the consensus reaching model is summarized as follows.

Let $t^{*}=t$. Output the final subgroups' decision matrices $R^{k\left(t^{*}\right)}(k=1,2, \ldots, n)$ and the final group decision $\operatorname{matrix} R^{c\left(t^{*}\right)}$.

The process of consensus reaching model can be simply described in Figure 1.

\section{Case Study}

In this section, an example of emergency decision-making problem in flood disaster is applied to indicate the feasibility of the presented method.

4.1. Case Background. There is a flood disaster hit $\mathrm{Hu}$ Nan Province, a south city in China, on July 3, 2018. After the flood disaster, the government carried out an emergency scheme based on instructions. As shown in Table 1, four preliminary plans were rapidly drawn up:

Twenty experts $E=\left(e_{1}, e_{2}, \ldots, e_{20}\right)$ from different fields were asked to make decisions based on these four alternatives $X=\left(x_{1}, x_{2}, x_{3}, x_{4}\right)$. We consider three criteria for each alternative: (1) personnel security rate $\left(f_{1}\right)$, the scale of evaluation value of personnel security rate is 0 to 1 ; (2) personnel injured rate $\left(f_{2}\right)$, the scale of the evaluation value of the effectiveness of equipment is the same to personnel security rate; (3) the development of situation of flood disaster $\left(f_{3}\right)$.

4.2. The Process of Group Decision-Making. We set that the scale of evaluation value of development situation of flood disaster is 1 to 100. Each decision-maker opinion should be seriously taken into account. Suppose that there is no conflict of interest among the decision-makers. In order to obtain the best alternative(s), the following steps need to be performed.

Step 1. Cluster the initial normalized individual decision matrices.

To save space, the normalized individual decision matrices are omitted. Base on the clustering method, which is described in Algorithm 1, the clustering threshold is set as $\zeta=0.8$, and the group can be divided into several smaller clusters. Table 2 shows the results, indicating that the original decision group can be divided into five clusters.

Step 2. Calculate the group decision matrix.

Aggregate the decision-makers' decision matrices into the subgroups' decision matrices by the (5). The weight of subgroup is calculated by the (2), and the group decision matrix is calculated by the individual decision matrices and the weight of cluster, which is adopted as

$$
R^{c(0)}=\left[\begin{array}{lll}
0.5099 & 0.8063 & 0.5721 \\
0.6400 & 0.4345 & 0.3138 \\
0.2837 & 0.7329 & 0.2879 \\
0.4810 & 0.1408 & 0.7346
\end{array}\right] .
$$

Step 3. Consensus measure and calculate the consensus threshold.

Compute the subgroup consensus levels by the (5), that is, $C I\left(R^{1(0)}\right)=0.7949, C I\left(R^{2(0)}\right)=0.7388, C I$ $\left(R^{3(0)}\right)=0.6488, C I\left(R^{4(0)}\right)=0.7263$ and $C I\left(R^{5(0)}\right)$ $=0.6141$. The initial group consensus level can be 
Input: the normalized individual decision matrices $R^{j(0)}(j=1,2, \cdots M)$, the subjective consensus threshold $\overline{L G C I}_{\text {sub }}$.

Output: the final group decision matrix $R^{c\left(t^{*}\right)}$.

Step 1. Large groups are divided into subgroups

Use the clustering method (Algorithm 1) described in Section 2.2 to classify the large group. Suppose, we get clusters $C=\left\{C^{1}, C^{2}, \ldots, C^{Y}\right\}$.

Step 2. Calculate the group decision matrix.

By applying (4), the decision matrices of subgroups can be normalized and aggregated into the group decision matrix.

Step 3. Determine the $\overline{L G C I}$.

The subjective $\overline{L G C I}_{\text {sub }}$ result and objective $\overline{L G C I}_{o b}$ results can be adopted to calculate $\overline{L G C I}$. By combining the number of decision-makers and the actual decision problems, the adjustment coefficient is determined, and this step can be removed in the following iteration.

Step 4. Calculate consensus

By using (5) to calculate the consensus level between each subgroup's decision matrices and the group decision matrix, that is, $C I\left(R^{l(t)}\right)(l=1,2, \ldots, Y)$. Then, the group consensus level can be obtained by applying (7). If $L G C I^{(t)} \geq \overline{L G C I}$, and proceed to Step 6; otherwise, move on to the next step.

Step 5. Consensus reaching process.

(a) Detect the noncooperative behavior (s)

According to the three identification rules described in Section 3.1, the noncooperative subgroup can be identified.

(b) Manage the noncooperative behavior (s).

Using a treatment method that describe in section 3.2 to address the noncooperative subgroup according to the opinion matrix that decision-makers modify. Enter the next iteration and return Step 2.

Step 6. Output related decision information.

Let $t^{*}=t$. Output the final subgroups' decision matrices $R^{k\left(t^{*}\right)}(k=1,2, \ldots, n)$ and the final group decision matrix $R^{c\left(t^{*}\right)}$.

\section{Algorithm 2:}

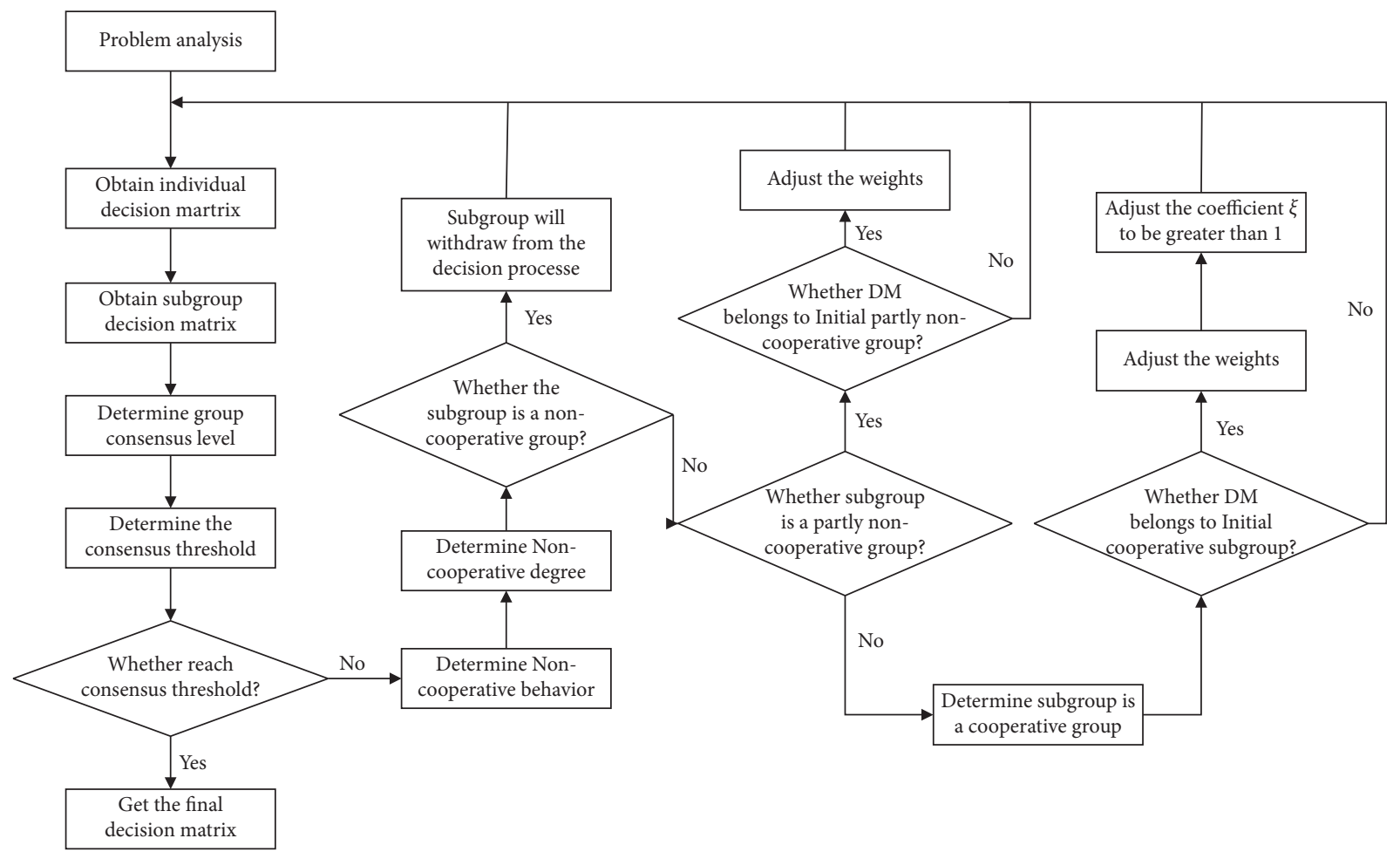

FIGURE 1: The process of consensus reaching model.

TABLE 1: Different selection strategies.

\begin{tabular}{cc}
\hline$x_{i}$ & Concrete measure \\
\hline$x_{1}$ & Find out trapped people and evacuate the seriously injured from the disaster areas to avoid further damage caused from flood disaster \\
$x_{2}$ & Treat the injured and stop searching for trapped people until the rescue equipment arrived \\
$x_{3}$ & Search for trapped people and treat the seriously injured in situ \\
$x_{4}$ & Search for trapped people and cease treating the injured until the medical team arrived \\
\hline
\end{tabular}


TABLE 2: The information of subgroup.

\begin{tabular}{lccc}
\hline$C^{k}$ & $n_{k}$ & $e_{i}$ & $\lambda_{k}^{(0)}$ \\
\hline$C^{1}$ & 7 & $e_{1}, e_{6}, e_{7}, e_{11}, e_{13}, e_{17}, e_{19}$ & 0.3500 \\
$C^{2}$ & 5 & $e_{2}, e_{3}, e_{8}, e_{9}, e_{18}$ & 0.2500 \\
$C^{3}$ & 3 & $e_{4}, e_{10}, e_{20}$ & 0.1500 \\
$C^{4}$ & 4 & $e_{5}, e_{12}, e_{15}, e_{16}$ & 0.2000 \\
$C^{5}$ & 1 & $e_{14}$ & 0.0500 \\
\hline
\end{tabular}

calculated as $\operatorname{LGCI}\left(R^{c(0)}\right)=0.7416$. The consensus threshold can be calculated from the subjective and objective aspect, the subjective threshold is set as 0.8 , and the objective threshold is 0.7416 . The adjustment coefficient is obtained by the (11), which is expressed as 0.2234. Finally, the consensus threshold is computed as 0.7869. Because of $\operatorname{LGCI}\left(R^{c(0)}\right)=0.7416<0.7869$, the consensus process should be applied to change some opinions.

Step 4. Consensus reaching process.

(i) First consensus reaching iteration

The detection object of the noncooperative cluster is determined by (12) and (13), and the calculation result shows that the subgroup $\mathrm{C}^{5}$ is the detection object. The decision-makers in the subgroup $\mathrm{C}^{5}$ modify their initial decision opinions to reach the consensus, the decision-makers who needed to reconsider their preferences were determined, and the modification criterion of the decision-maker is the group decision matrix. The decision matrix of decision-maker $A^{j(1)}$ can be obtained after modification. The temporary subgroup decision matrix $R^{\prime 5(1)}$ can be computed by the decision matrix of decision-maker $A^{j(1)}$. The consensus level of clusters is calculated by the temporary subgroup decision matrix $R^{\prime 5(1)}$, which is expressed as $C I^{\prime}\left(R^{5(1)}\right)$. Comparing the temporary subgroup consensus level $C I^{\prime}\left(R^{5(1)}\right)$ with consensus level $C I\left(R^{5(0)}\right)$, the result shows that the $C I^{\prime}\left(R^{5(1)}\right)$ is less than $C I\left(R^{5(0)}\right)$, and the subgroup $C^{5}$ is noncooperative subgroup, which denotes that the subgroup $\mathrm{C}^{5}$ expressed the high noncooperative behavior.

Based on the noncooperative management method, $\phi_{l^{*}}^{(t)} \leq 0$, the subgroup $C^{5}$ is suggested to quit the consensus process. Thus, the number of subgroups is null, and the decision matrix is 0 , and there are four subgroups by clustering the decision-maker after management. Because of the change of subgroup number, the weight of cluster should be recalculated by (2), which is described as $\lambda^{(1)}=(0.350 .3500,0.2500$, $0.2000,0.2000,0)$. Continuing consensus measure, the new cluster consensus levels are $C I\left(R^{1(1)}\right)=0.7914$, $C I\left(R^{2(1)}\right)=0.7425, \quad C I\left(R^{3(1)}\right)=0.7259$ and $C I$ $\left(R^{4(1)}\right)=0.7238$. The group consensus level is $L G C I$ $\left(R^{c(1)}\right)=0.7526<0.7869$. Thus, the consensus reaching process continues.

(ii) Second consensus reaching iteration
As $C I\left(R^{4(1)}\right)=\min \left\{C I\left(R^{i(1)}\right) \mid i=1,2,3,4\right\}$, the cluster $\mathrm{C}^{4}$ can be regarded as detection object in the 2 -th round iterations, and the decision-makers in the cluster $\mathrm{C}^{4}$ are required to modify their own opinion. The decision-maker's decision opinion in the 2-th rounds can be obtained after discussion, which is described as $A^{j(2)}$, aggregating the individual decision matrices, obtaining the temporary group decision matrix. The temporary consensus level is determined by the temporary subgroup decision matrix $R^{\prime 4(2)}$ and temporary group decision matrix. Comparing the temporary subgroup consensus level $C I^{\prime}\left(R^{4(2)}\right)$ with consensus level $C I\left(R^{4(1)}\right)$, the result shows that the $C I^{\prime}\left(R^{4(2)}\right)$ is more than $C I\left(R^{4(1)}\right)$, but only part of decisionmakers in subgroup $C^{4}$ are willing to modify their opinions; thus, the subgroup $\mathrm{C}^{4}$ is noncooperative subgroup.

The conflict degree of subgroup $\mathrm{C}^{4}$ is calculated to denote the decision-makers who modify their decision opinion. Based on the result of the calculation, there are four decision-makers in the subgroup $\mathrm{C}^{4}$ who are willing to modify their decision opinions. The noncooperative degree of subgroup is computed as $0.5<\phi_{l^{*}}^{(t)} \leq 1$. According to (20), whether the decision-maker who modifies his opinion belongs to the initial subgroup can be judged. The result shows that the decision-maker $e_{5}$ does not belong to the subgroup $\mathrm{C}^{4}$ after changing their initial decision opinion by the (21), and the decision-makers $e_{15}$, $e_{16}$ belong to the initial subgroup $\mathrm{C}^{4}$. The decision-maker $e_{5}$ is clustered by the Algorithm 1; the gathered degree between the decision-maker $e_{5}$ and the subgroup $\mathrm{C}^{1}$ is $0.9135>0.8$; thus, the decision-maker $e_{5}$ belongs to the subgroup $C^{1}$. The weights of $C^{4}$ and $C^{1}$ are updated according to equation (2) and the number of decisionmakers. The new subgroup weight can be denoted as $\lambda^{(2)}=(0.4517,0.2438,0.1976,0.1069)$.

The new subgroup consensus levels are $C I\left(R^{1(2)}\right)=$ 0.8014, $C I\left(R^{2(2)}\right)=0.7956, C I\left(R^{1(2)}\right)=0.7643$ and $C I$ $\left(R^{1(2)}\right)=0.7234$. The group consensus level is $\operatorname{LGCI}\left(R^{c(2)}\right)$ $=0.7901>0.7869$. After two iterations, the decision-makers obtain consistency, and the final group consensus level meets the predefined requirement.

Due to the reaching of consensus threshold by group consensus level, the group decision matrix can be used to determine, which alternative is optimal, and the final calculation result of a group decision is expressed as

$$
R^{c(2)}=\left[\begin{array}{lll}
0.5119 & 0.8313 & 0.5596 \\
0.6358 & 0.4491 & 0.2745 \\
0.2753 & 0.6829 & 0.3379 \\
0.4859 & 0.1408 & 0.7529
\end{array}\right]
$$

According to the weight of the attribute, the decision vector is calculated as $(0.6343,0.4531,0.4321,0.4599)$, and the value of alternative $x_{1}$ is 0.6343 . Thus, the alternative $x_{1}$ is the most optimal. 


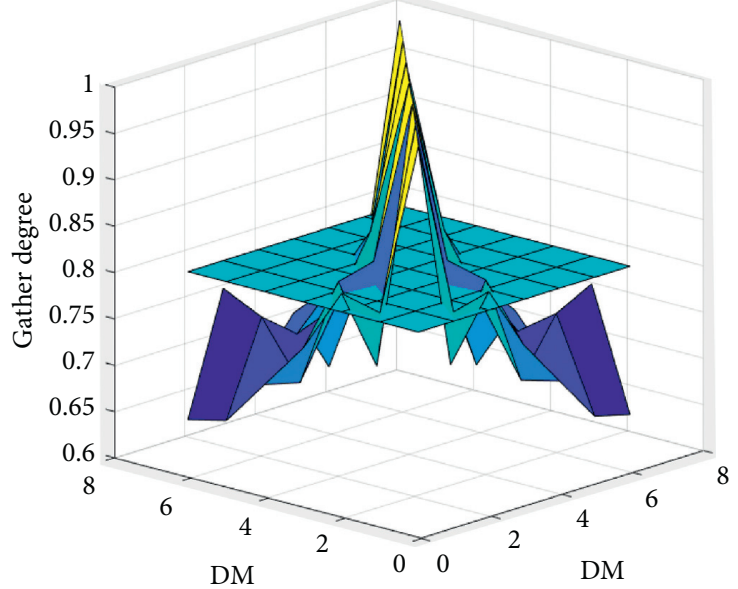

(a)

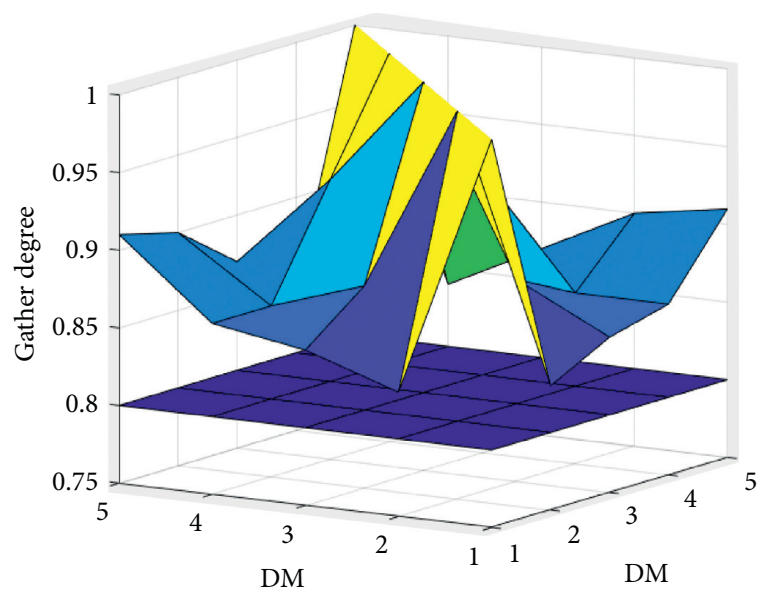

(b)

Figure 2: The effect of gather degree of decision-makers of different clustering methods. (a) The gather degree of traditional clustering method. (b) The gathered degree of improved clustering method.

\section{Discussion}

In this paper, we proposed a novel method to calculate the consensus threshold from the subjective and objective aspect. It is unlike the traditional determination method consensus threshold. Based on this consensus threshold and the traditional clustering method, the improved clustering method is developed. The improved method depends on the similarities between the decision-makers' decision opinions and subgroups' decision opinion. To better reflect the advantages of the clustering method, the case in Section 3 is adopted to compare the difference between the current clustering method and the traditional clustering method. We take the subgroup that includes the decision-maker $e_{1}$ as example, and the gathered degree of decision-makers for different clustering methods can be computed. And comparing the clustering thresholds, the effect of gather degree is shown in Figure 2.

From Figure 2, we can obtain that there are many decision-makers whose gathered degree is less than clustering threshold for the traditional clustering method (see Figure 2(a)); but it can be seen from Figure 2(b) that the gathered degree for any decision-makers is more than 0.8 after using the improved clustering method. The decisionmakers using the improved method have higher gather degree than those using the traditional method. The result shows that the traditional clustering method considers the whole gather degree and omits the gather degree among decision-makers. Thus, the improved clustering method is more suitable to divide into the group.

Except for one group comparison, it can also compare the composition of different subgroups and the degree of subgroup clustering. The results of the comparison can be represented in Figure 3.

In Figure 3, groups are more concentrated. And compared to using traditional methods, each group has a higher degree of clustering after using the improved method in this article. The goal of MALGDM is to achieve a high degree of consistency. And the higher degree of clustering represents that the large group has higher consensus level. Thus, the method used in this article is more effective than the traditional one in dealing with MALGDM problem.

Due to adherence to the principle that noncooperative behavior needs full consideration, the group consensus level increased from 0.7416 to 0.7901 , which means that the management of the noncooperative behavior is helpful in reaching the consensus level. Generally, the noncooperative behavior can be addressed by two steps: the detection of the noncooperative behavior and the management of the noncooperative behavior. The current detection method uses the subjective adjustment result of subgroup to detect the noncooperative behavior. Unlike the current detection method, the practical adjustment result of subgroup is adopted to judge the noncooperative behaviors, that is, comparing the change degree of the subgroup decision matrix in the $t$-th rounds and $t-1$-th rounds.

For the management of the noncooperative behavior, the novel approach is explored to manage the noncooperative behavior. In general, the weight of the subgroup can be adjusted to manage the noncooperative behavior, and the decision matrix is transformed by the adjustment coefficient. In the practical decision problem, the adjustment opinion which the decision-maker is willing to accept should be respected. Thus, the noncooperative behavior is managed by adjusting the weight of subgroup, recalculating the subgroup decision matrix in this paper. In this study, the subgroups are allowed to modify. Generally, the number of subgroups is changed due to an enforced exit rule; nevertheless, the subgroups themselves remained the same. It is assumed that the decision makers can choose to modify their opinions under discussion, which makes it sensible that the subgroups they are classified into may also change. The example in Section 4 validated this point. Meanwhile, the weight of subgroup can be adjusted by the contribution of the cluster in the group consensus level and the number of the decisionmakers who are willing to modify their initial opinion in the cluster. 


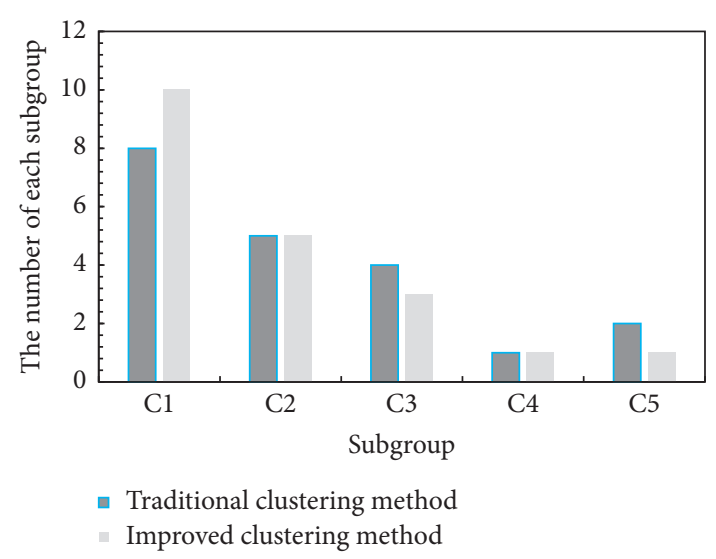

(a)

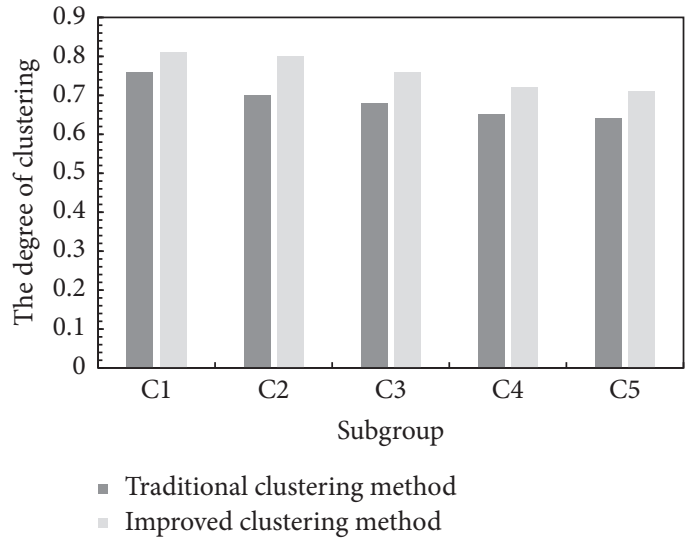

(b)

Figure 3: Comparisons between different groups under two different methods. (a) The number of each subgroup under two different methods. (b) The gathered degree of each subgroup under two different methods.

Different decision-making models have different emphasis. Therefore, there is no model that can be referred to as the best. Despite offered valuable methods to handle multiattribute large group decision-making problems, there are limitations in the proposed model: it is not easy to determine the subjective consensus threshold in this paper. Similar to other consensus models, the subjective consensus threshold needs to be decided by the moderator or group. Although empirical values for the consensus threshold can be given, the determination of the subjective consensus threshold depends on the actual problems and simulations. The subjective consensus threshold makes it free for the moderator and/or the group to grasp the decision processes.

\section{Conclusion}

The MALGDM problem becomes more and more significant for participants and stakeholders to make a consensus-based decision. The main contributions of the paper are as follows:

(1) A novel clustering method was adopted to divide the large group into several clusters. The similarity is calculated to express the gather degree of decisionmakers, and the decision-maker can be classified by the gathered degree. The value of gathered degree decides the number of subgroups.

(2) A consensus framework for the consensus reaching process in a MALGDM is proposed. The consensus threshold is determined by the consensus level and subjective consensus threshold. Meanwhile, the noncooperative behavior of decision-makers is determined, and the subgroup that includes the decision-maker who expresses the noncooperative behavior is defined as the noncooperative subgroup.

(3) A novel consensus reaching process is designed to address the noncooperative subgroup. The noncooperative subgroup is detected by the number of decision-makers and the consensus level, and by determining three noncooperative behavior situations. The weight of the subgroup is adjusted to reach the consensus level, and the subgroups in our proposed approach are allowed to change. Thus, there are three approaches to manage the noncooperative behavior: adjustment weight of subgroup, quitting the decision process, and changing the subgroups in each interactive round.

Further, some other clustering methods can be incorporated in the proposal to detect the influences of clustering on model convergence. For the classification of large groups, it may be a good alternative to adopt an automatic feedback strategy such as an optimization-based approach. However, some limitations exist in the research. Due to the difference of risk preference, decision-makers may make decisions that are difficult to coordinate, and this paper does not cover the psychological perception of decision makers, which may be an important research direction in LGDM problem in the future. Meanwhile, there are many factors that can influence the decision-making, and the interactions of factors may influence the results. Thus, the approach considering the interactions of factors will be used to explore multiattribute interactions of LGDM.

\section{Data Availability}

The data used to support the findings of this study are included within the article.

\section{Conflicts of Interest}

The authors declare that they have no conflicts of interest.

\section{Acknowledgments}

The authors would like to appreciate the support of the students and staff of the Hubei Key Laboratory of Construction and Management in Hydropower Engineering for their assistance in research. The research was supported by National Natural Science Foundation of China (Grant No. 51878385) and Natural Science Research Project of Yichang (Grant No. A21-3-001). 


\section{References}

[1] Y. K. Hu, S. H. Yu, J. J. Chu et al., "A consensus-reaching approach to the evaluation of product design alternatives with multiple preference structures," Computational Intelligence and Neuroscience, vol. 2021, Article ID 6992648, 16 pages, 2021.

[2] P. Ren, Z. Xu, and H. Liao, "Intuitionistic multiplicative analytic hierarchy process in group decision making," Computers \& Industrial Engineering, vol. 101, pp. 513-524, 2016.

[3] C. Hou, Y. Wen, Y. He et al., "Public stereotypes of recycled water end uses with different human contact: evidence from event-related potential (ERP)," Resources, Conservation and Recycling, vol. 168, Article ID 105464, 2021.

[4] B. Cheng, K. Lu, J. Li, H. Chen, X. Luo, and M. Shafique, "Comprehensive assessment of embodied environmental impacts of buildings using normalized environmental impact factors," Journal of Cleaner Production, vol. 334, Article ID 130083, 2022.

[5] H. Li, Y. Cao, L. Su, and Q. Xia, "An interval pythagorean fuzzy multi-criteria decision making method based on similarity measures and connection numbers," Information, vol. 10, no. 2, p. 80, 2019.

[6] F. Liu, J. W. Zhang, Q. Yu, Y. N. Peng, and W. Pedrycz, "On weak consistency of interval additive reciprocal matrices," Fuzzy Optimization and Decision Making, vol. 2020, no. 19, 175 pages, 2020.

[7] E. Abad-Segura, M.-D. González-Zamar, E. López-Meneses, and E. Vázquez-Cano, "Financial technology: review of trends, approaches and management," Mathematics, vol. 8, no. 6, 951 pages, 2020.

[8] N. M. Scala, J. Rajgopal, L. G. Vargas, and K. L. Needy, “Group decision making with dispersion in the analytic hierarchy process," Group Decision and Negotiation, vol. 25, no. 2, pp. 355-372, 2016.

[9] S. Cali and S. Y. Balaman, "A novel outranking based multi criteria group decision making methodology integrating ELECTRE and VIKOR under intuitionistic fuzzy environment," Expert Systems with Applications, vol. 119, pp. 36-50, 2019.

[10] J. Tang, F. Meng, F. J. Cabrerizo, and E. Herrera-Viedma, “A procedure for group decision making with interval-valued intuitionistic linguistic fuzzy preference relations," Fuzzy Optimization and Decision Making, vol. 18, no. 4, pp. 493-527, 2019.

[11] X.-B. Mao, M. Wu, J.-Y. Dong, S.-P. Wan, and Z. Jin, “A new method for probabilistic linguistic multi-attribute group decision making: application to the selection of financial technologies," Applied Soft Computing, vol. 77, pp. 155-175, 2019.

[12] J. Chu, X. Liu, Y. Wang, and K.-S. Chin, “A group decision making model considering both the additive consistency and group consensus of intuitionistic fuzzy preference relations," Computers \& Industrial Engineering, vol. 101, pp. 227-242, 2016.

[13] A. Mahmoudi, S. Sadi-Nezhad, and A. Makui, "A hybrid fuzzy-intelligent system for group multi-attribute decision making," International Journal of Fuzzy Systems, vol. 18, no. 6, pp. 1117-1130, 2016.

[14] R. Krishankumar, K. S. Ravichandran, M. I. Ahmed, S. Kar, and X. D. Peng, "Interval-Valued probabilistic hesitant fuzzy set based muirhead mean for multi-attribute group decisionmaking," Mathematics, vol. 7, no. 4, p. 342, 2019.
[15] M. Przybyła-Kasperek and A. Wakulicz-Deja, "Global decision-making system with dynamically generated clusters," Information Sciences, vol. 270, pp. 172-191, 2014.

[16] A. Azadeh, M. Saberi, and M. Anvari, “An Integrated Artificial Neural Network Fuzzy C-Means-Normalization Algorithm for performance assessment of decision-making units: the cases of auto industry and power plant," Computers \& Industrial Engineering, vol. 60, no. 2, pp. 328-340, 2011.

[17] L. Peide and L. Ying, "An improved failure mode and effect analysis method for multi-criteria group decision-making in green logistics risk assessment," Reliability Engineering \& System Safety, vol. 215, Article ID 107826, 2021.

[18] Y. Xue, Y. Deng, and H. Garg, "Uncertain database retrieval with measure - based belief function attribute values under intuitionistic fuzzy set," Information Sciences, vol. 546, pp. 436-447, 2021.

[19] Y. Li, H. Garg, and Y. Deng, "A new uncertainty measure of discrete Z-numbers," International Journal of Fuzzy Systems, vol. 22, no. 3, pp. 760-776, 2020.

[20] A. Tapia-Rosero, A. Bronselaer, and G. De Tré, “A method based on shape-similarity for detecting similar opinions in group decision-making," Information Sciences, vol. 258, pp. 291-311, 2014.

[21] X. Xu, B. Wang, and Y. Zhou, "A method based on trust model for large group decision-making with incomplete preference information," Journal of Intelligent and Fuzzy Systems, vol. 30, no. 6, pp. 3551-3565, 2016.

[22] C.-g. Cai, X.-h. Xu, P. Wang, and X.-h. Chen, “A multi-stage conflict style large group emergency decision-making method," Soft Computing, vol. 21, no. 19, pp. 5765-5778, 2017.

[23] F. Meng, Q. An, and X. Chen, "A consistency and consensusbased method to group decision making with interval linguistic preference relations," Journal of the Operational Research Society, vol. 67, no. 11, pp. 1419-1437, 2016.

[24] X.-h. Xu, X.-y. Zhong, X.-h. Chen, and Y.-j. Zhou, "A dynamical consensus method based on exit-delegation mechanism for large group emergency decision making," Knowledge-Based Systems, vol. 86, pp. 237-249, 2015.

[25] M. Akram, A. Adeel, and J. C. R. Alcantud, "Multi-criteria group decision-making using an m-polar hesitant fuzzy TOPSIS approach,” Symmetry, vol. 11, no. 6, p. 795, 2019.

[26] M. Zhao, X.-y. Ma, and D.-w. Wei, "A method considering and adjusting individual consistency and group consensus for group decision making with incomplete linguistic preference relations," Applied Soft Computing, vol. 54, pp. 322-346, 2017.

[27] H. Wang, Y. Ju, and P. Liu, "Multi-attribute group decisionmaking methods based on q-rung orthopair fuzzy linguistic sets," International Journal of Intelligent Systems, vol. 34, no. 6, pp. 1129-1157, 2019.

[28] M. Riaz and S. T. Tehrim, "Multi-attribute group decision making based on cubic bipolar fuzzy information using averaging aggregation operators," Journal of Intelligent and Fuzzy Systems, vol. 37, no. 2, pp. 2473-2494, 2019.

[29] F. Meng, S.-M. Chen, and J. Tang, "Group decision making based on acceptable multiplicative consistency of hesitant fuzzy preference relations," Information Sciences, vol. 524, no. 524, pp. 77-96, 2020.

[30] L. Martínez and J. Montero, "Challenges for improving consensus reaching process in collective decisions," New Mathematics and Natural Computation, vol. 2, no. 3, pp. 203-217, 2007.

[31] I. J. Perez, F. J. Cabrerizo, S. Alonso, and E. Herrera-Viedma, "A new consensus model for group decision making problems with non-homogeneous experts," IEEE Transactions on 
Systems, Man, and Cybernetics: Systems, vol. 44, no. 4, pp. 494-498, 2014.

[32] Y. Xu, X. Wen, and W. Zhang, "A two-stage consensus method for large-scale multi-attribute group decision making with an application to earthquake shelter selection," Computers \& Industrial Engineering, vol. 116, pp. 113-129, 2018.

[33] F. Jin, H. Garg, L. Pei, J. Liu, and H. Chen, "Multiplicative consistency adjustment model and data envelopment analysis-driven decision-making process with probabilistic hesitant fuzzy preference relations," International Journal of Fuzzy Systems, vol. 22, no. 7, pp. 2319-2332, 2020.

[34] Y. Dong, H. Zhang, and E. Herrera-Viedma, "Integrating experts' weights generated dynamically into the consensus reaching process and its applications in managing non-cooperative behaviors," Decision Support Systems, vol. 84, pp. 1-15, 2016.

[35] Y. Dong, S. Zhao, H. Zhang, F. Chiclana, and E. HerreraViedma, "A self-management mechanism for noncooperative behaviors in large-scale group consensus reaching processes," IEEE Transactions on Fuzzy Systems, vol. 26, no. 6, pp. 3276-3288, 2018.

[36] I. Palomares, L. Martinez, and F. Herrera, “A consensus model to detect and manage noncooperative behaviors in large-scale group decision making," IEEE Transactions on Fuzzy Systems, vol. 22, no. 3, pp. 516-530, 2014.

[37] Z. Wu and J. Xu, "A consensus model for large-scale group decision making with hesitant fuzzy information and changeable clusters," Information Fusion, vol. 41, pp. 217-231, 2018.

[38] F. J. Quesada, I. Palomares, and L. Martínez, "Managing experts behavior in large-scale consensus reaching processes with uninorm aggregation operators," Applied Soft Computing, vol. 35, pp. 873-887, 2015.

[39] S. Nazari, A. Ahmadi, S. Kamrani Rad, and B. Ebrahimi, "Application of non-cooperative dynamic game theory for groundwater conflict resolution," Journal of Environmental Management, vol. 270, Article ID 110889, 2020.

[40] X.-h. Xu, Z.-j. Du, and X.-h. Chen, "Consensus model for multi-criteria large-group emergency decision making considering non-cooperative behaviors and minority opinions," Decision Support Systems, vol. 79, pp. 150-160, 2015.

[41] P. Mandal, S. Samanta, and M. Pal, "Large-scale Group Decision-Making Based on Pythagorean Linguistic Preference Relations Using Experts Clustering and Consensus Measure with Non-cooperative Behavior Analysis of Clusters," Complex \& Intelligent Systems, 2021.

[42] B. Liu, Y. Shen, X. Chen, Y. Chen, and X. Wang, "A partial binary tree DEA-DA cyclic classification model for decision makers in complex multi-attribute large-group interval-valued intuitionistic fuzzy decision-making problems," Information Fusion, vol. 18, pp. 119-130, 2014.

[43] Y. Liu, Z.-P. Fan, and Y. Zhang, "A method for stochastic multiple criteria decision making based on dominance degrees," Information Sciences, vol. 181, no. 19, pp. 4139-4153, 2011.

[44] Q. Dong and T. L. Saaty, "An analytic hierarchy process model of group consensus," Journal of Systems Science and Systems Engineering, vol. 23, no. 3, pp. 362-374, 2014.

[45] Y. Dong, Z.-P. Fan, and S. Yu, "Consensus building in a local context for the AHP-GDM with the individual numerical scale and prioritization method," IEEE Transactions on Fuzzy Systems, vol. 23, no. 2, pp. 354-368, 2015.

[46] D. Ben-Arieh and Z. F. Zhifeng Chen, "Linguistic-labels aggregation and consensus measure for autocratic decision making using group recommendations," IEEE Transactions on Systems, Man, and Cybernetics-Part A: Systems and Humans, vol. 36, no. 3, pp. 558-568, 2006. 